\title{
A Finite Element Method Using Singular Functions: Interface Problems
}

\author{
Seokchan Kim* ${ }^{*}$ Zhiqiang Cai ${ }^{\dagger} \quad$ Jae-Hong Pyo ${ }^{\ddagger}$ Sooryoun Kong*
}

\begin{abstract}
The solution of the interface problem is only in $H^{1+\alpha}(\Omega)$ with $\alpha>0$ possibly close to zero and, hence, it is difficult to be approximated accurately. This paper studies an accurate numerical method on quasi-uniform grids for two-dimensional interface problems. The method makes use of a singular function representation of the solution, dual singular functions, and an extraction formula for stress intensity factors. Using continuous piecewise linear elements on quasi-uniform grids, our finite element approximation is shown to be optimal, $O(h)$, accurate in the $H^{1}$ norm. This is confirmed by numerical experiments for interface problems with $\alpha<0.1$. An $O\left(h^{1+\alpha}\right)$ error bound in the $L^{2}$ norm is also established by the standard duality argument. For small $\alpha$, this improvement over the $H^{1}$ error bound is negligible. However, numerical tests presented in this paper indicate that the $L^{2}$ norm accuracy is much better than the theoretical error bound.
\end{abstract}

Key words. Interface singularity, Finite Element, Singular function, Stress intensity factor

AMS(MOS) subject classifications. 65F10, 65F30

\section{Introduction}

Let $\Omega$ be an open, bounded polygonal domain in $\mathbf{R}^{2}$. Consider the following model interface problem

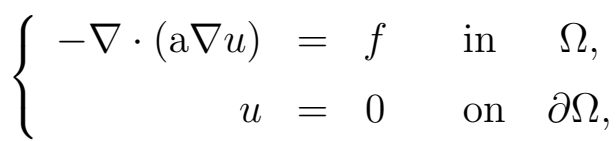

*Department of Applied Mathematics, Changwon National University, Changwon, 641-773, Republic of Korea (sckim@changwon.ac.kr,ksr@changwon.ac.kr). The author was partially supported by KOSEF 2002-5-103-01-2

$\dagger$ Department of Mathematics, Purdue University, 1395 Mathematical Science Building, West Lafayette, IN 47907-1395 (zcai@math.purdue.edu). The author was sponsored in part by the National Science Foundation under grant DMS-0511430.

¥This study was supported by 2007 Research Grant from Kangwon National University. (jhpyo@kangwon.ac.kr) 
where the diffusion coefficient $\mathrm{a}(x)$ is a given piecewise constant function and $f$ is a given function in $L^{2}(\Omega)$. (Here and thereafter we use the standard notation and definition of the Sobolev spaces.)

It is well-known that the solution of (1.1) is in $H^{1+\alpha}(\Omega)$ with $\alpha>0$ possibly close to zero (see [15]). Such low regularity of the solution makes lower order finite element approximations on quasi-uniform grids inaccurate. There were several approaches in the literatures for overcoming this difficulty, for example, local mesh refinement (see $[1,19])$ and the $p($ or $h p$ ) version of the finite element method (see $[20,21]$ ). To achieve optimal convergence of lower order finite elements on quasi-uniform grids, a class of finite element methods studied by various researchers is to make use of the singular function representation of the solution. This includes the singular function method (see, e.g., $[10,8]$ ), the dual singular function method (see $[9,2,3]$ ), and the multigrid version of the dual singular function method (see [4]).

Solutions of many partial differential equations have singular function representations: a decomposition as the sum of regular and singular parts of the solution. Moreover, the singular part has an explicit form and the unknown coefficient of the singular part, the so-called stress intensity factor, is given by the extraction formula depending on the given data and the original solution. The singular and dual singular function methods augment approximation spaces by using the singular and/or dual singular functions. As an alternative, in this paper we develop, analyze, and test an accurate finite element method that also uses both the singular and dual singular functions. Differing from other methods of this type, our method aims for approximation of the regular part of the solution that is much smoother than the solution itself. Once the regular part of the solution is computed, then the stress intensity factors and the solution itself can be calculated with negligible cost and without degrading accuracy. So the key of our method is to derive a well-posed and smoother problem for the regular part of the solution. This is done by developing a new extraction formula for the stress intensity factors in terms of the regular part of the solution, which is similar to but more complicated than our previous work [6] for Poisson problems with corner singularities. Since this new problem is $H^{2}$ regular in each sub-domain, we show that the standard continuous piecewise linear finite element approximation on quasi-uniform grids has optimal, $O(h)$, accuracy in the $H^{1}$ norm. This, in turn, implies that approximations to the stress intensity factors and the original solution are $O(h)$ accurate in the absolute value and the $H^{1}$ norm, respectively. This theoretical prediction is confirmed by numerical results for interface problems in $H^{1+\alpha}$ with $\alpha<0.1$ and with singular points at either corner or interior.

To establish the $L^{2}$ norm error bound, we adapt the standard duality argument. In order to achieve an extra order accuracy in the $L^{2}$ norm, it requires that the properly chosen adjoint problem has the full $H^{2}$ regularity and yields the $L^{2}$ norm of the error. At this stage, we are unable to find an adjoint problem satisfying both the requirements. Hence, we use an adjoint problem with a simplified right-hand side that naturally gives the $L^{2}$ norm of the error but has only $H^{1+\alpha}$ regularity. With this choice, we can only prove $O\left(h^{1+\alpha}\right)$ error bound in the $L^{2}$ norm. For small $\alpha>0$, obviously this theoretical 
error bound improves only a little over the $H^{1}$ norm error bound. Since the regular part of the solution is piecewise $H^{2}$, it is possible that the approximation accuracy in the $L^{2}$ norm is much better than the theoretical bound. One of the purposes of this paper is to demonstrate numerically that this is indeed true.

The paper is organized as follows. Section 2 introduces the two-dimensional interface problem, its singular function representation of the solution, and an extraction formula for the stress intensity factors. Section 3 derives the variational problem for the regular part of the solution via a newly developed extraction formula and establishes its well-posedness by the Fredholm alternative. Section 4 introduces the finite element method and estimates error bounds in both the $H^{1}$ and $L^{2}$ norms. Finally, numerical results are presented in Section 5.

\section{Interface Singularities}

Let $\Omega_{j}(j=1, \ldots, J)$ be open, polygonal subdomains of $\Omega$ and let $\left\{\Omega_{j}\right\}_{j=1}^{J}$ be a partition of the domain $\Omega$

$$
\Omega_{i} \cap \Omega_{j}=\emptyset \quad \text { for } i \neq j \quad \text { and } \quad \bigcup_{j=1}^{J} \bar{\Omega}_{j}=\bar{\Omega} \text {. }
$$

Let $\Omega_{\Sigma}=\bigcup_{j=1}^{J} \Omega_{j}$. Assume that the diffusion coefficient a is piecewise constant with respect to the partition

$$
\mathrm{a}(x)=\mathrm{a}_{j}>0 \quad \text { in } \Omega_{j}
$$

for $j=1, \ldots, J$. Let $\Gamma_{i j}=\partial \Omega_{i} \cap \partial \Omega_{j}$ denote the common edge of $\Omega_{i}$ and $\Omega_{j}$ and let $\mathbf{n}_{j}$ be the outward unit normal vector to the boundary $\partial \Omega_{j}$ of $\Omega_{j}$. Then problem (1.1) can be rewritten as: find $u \in H_{0}^{1}(\Omega)$ such that

$$
-\mathrm{a}_{j} \Delta u=f \quad \text { in } \Omega_{j}
$$

for $j=1, \ldots, J$ with interface conditions

$$
\left.\mathrm{a}_{i} \frac{\partial u}{\partial \mathbf{n}_{i}}\right|_{\Gamma_{i j}}+\left.\mathrm{a}_{j} \frac{\partial u}{\partial \mathbf{n}_{j}}\right|_{\Gamma_{i j}}=0
$$

for $i, j=1, \ldots, J$ such that $\Gamma_{i j} \neq \emptyset$ (see [13]).

For simplicity of presentation, assume that there is only one interface vertex $p$ located at the origin. Extension to the domain with a finite number of reentrant corners [6] and interface vertices is straightforward. For interface problems, it is well-known that the solution has a singular function representation (see $[13,14,15,16,17]$ ).

Let $\Omega_{m_{1}}, \Omega_{m_{2}}, \ldots, \Omega_{m_{I}}$ be the subdomains sharing $p$ as a common vertex. Let $\delta>0$ be a small number such that $p$ is the only vertex of the subdomians inside the disc $D(p, \delta)$ centered at $p$ with radius $\delta$. When $p$ belongs to the boundary of the domain $\Omega$, let polar coordinates $(r, \theta)$ be chosen so that $D(p, \delta) \cap \Omega_{m_{i}}=\left\{(r, \theta): 0<r<\delta, \omega_{i-1}<\right.$ 
$\left.\theta<\omega_{i}\right\}$ for $1 \leq i \leq I$, where $\omega_{0}=0$, and $\omega_{I}=\omega$ be the angle between the two edges of $\partial \Omega$ emanating from $p$. When $p$ belongs to the interior of the domain $\Omega$, the subdomians $\left\{\Omega_{m_{i}}\right\}_{i=1}^{I}$ completely surround $p$. So we may have the polar coordinates such that $\omega_{0}=0$ and $\omega_{I}=2 \pi$. Let $\lambda_{k}=\left(\alpha_{k}\right)^{2}$ and $\Theta_{k}(\theta)$ for $k \geq 1$ be, respectively, the positive eigenvalues and the corresponding eigenfunctions of the Sturm-Liouville problem at the vertex: in subintervals $\left(\omega_{i-1}, \omega_{i}\right)(i=1, \ldots, I)$

$$
-\Theta^{\prime \prime}(\theta)=\lambda \Theta(\theta)
$$

on interfaces $\omega_{i}(i=1, \ldots, I-1)$

$$
\lim _{\theta \rightarrow \omega_{i}^{-}} \Theta(\theta)=\lim _{\theta \rightarrow \omega_{i}^{+}} \Theta(\theta) \quad \text { and } \quad a_{m_{i}} \lim _{\theta \rightarrow \omega_{i}^{-}} \Theta^{\prime}(\theta)=a_{m_{i+1}} \lim _{\theta \rightarrow \omega_{i}^{+}} \Theta^{\prime}(\theta),
$$

and on boundaries $\theta=0, \theta=\omega$ or $2 \pi$

$$
\lim _{\theta \rightarrow 0^{+}} \Theta(\theta)=\lim _{\theta \rightarrow \omega^{-}} \Theta(\theta)=0 \quad \text { if } p \in \partial \Omega
$$

$$
\lim _{\theta \rightarrow 0^{+}} \Theta(\theta)=\lim _{\theta \rightarrow(2 \pi)^{-}} \Theta(\theta) \quad \text { and } \quad \mathrm{a}_{m_{1}} \lim _{\theta \rightarrow 0^{+}} \Theta^{\prime}(\theta)=\mathrm{a}_{m_{I}} \lim _{\theta \rightarrow(2 \pi)^{-}} \Theta^{\prime}(\theta) \quad \text { if } p \in \Omega,
$$

where the eigenfunctions are normalized as follows

$$
\sum_{i=1}^{I} \int_{\omega_{i-1}}^{\omega_{i}} \mathrm{a}_{m_{i}} \Theta_{j}(\theta) \Theta_{k}(\theta) d \theta=\delta_{j k}:= \begin{cases}1 & \text { if } j=k \\ 0 & \text { if } j \neq k\end{cases}
$$

Let $\alpha_{1} \leq \ldots \leq \alpha_{L}$ be all $\alpha_{l}^{\prime} s$ that satisfy $0<\alpha_{l}<1$. Define the singular functions and the dual singular functions by

$$
s_{l}(r, \theta)=r^{\alpha_{l}} \Theta_{l}(\theta) \quad \text { and } \quad s_{-l}(r, \theta)=r^{-\alpha_{l}} \Theta_{l}(\theta),
$$

respectively. Note that $s_{l}$ and $s_{-l}$ are twice differentiable and harmonic in each subdomain $\Omega_{j}\left(\Delta s_{l}=\Delta s_{-l}=0\right.$ in $\left.\Omega_{j}\right)$. It is easy to see that for $i=1, \ldots, I$

$$
s_{l} \in H^{1+\alpha_{l}-\varepsilon}\left(\Omega_{m_{i}}\right) \quad \text { and } \quad s_{-l} \in H^{1-\alpha_{l}-\epsilon}\left(\Omega_{m_{i}}\right)
$$

for any $\varepsilon>0$. On the interface $\Gamma_{m_{i} m_{i+1}}=\partial \Omega_{m_{i}} \cap \partial \Omega_{m_{i+1}}$, the second equation in (2.5) implies

$$
\begin{aligned}
\left.\mathrm{a}_{m_{i}} \frac{\partial s_{l}}{\partial \mathbf{n}_{m_{i}}}\right|_{\Gamma_{m_{i} m_{i+1}}}+\left.\mathrm{a}_{m_{i+1}} \frac{\partial s_{l}}{\partial \mathbf{n}_{m_{i+1}}}\right|_{\Gamma_{m_{i} m_{i+1}}}=0, \\
\left.\mathrm{a}_{m_{i}} \frac{\partial s_{-l}}{\partial \mathbf{n}_{m_{i}}}\right|_{\Gamma_{m_{i} m_{i+1}}}+\left.\mathrm{a}_{m_{i+1}} \frac{\partial s_{-l}}{\partial \mathbf{n}_{m_{i+1}}}\right|_{\Gamma_{m_{i} m_{i+1}}}=0 .
\end{aligned}
$$


Set

$$
B\left(r_{1} ; r_{2}\right)=\left\{(r, \theta): r_{1}<r<r_{2} \text { and } 0<\theta<\omega\right\} \cap \Omega \quad \text { and } B\left(r_{1}\right)=B\left(0 ; r_{1}\right) .
$$

Define a family of cut-off functions of $r$ for a fixed $\omega$ as follows

$$
\eta_{\rho}(r)= \begin{cases}1 & \text { in } B\left(\frac{\rho R}{2}\right), \\ \frac{15}{16}\left\{\frac{8}{15}-\left(\frac{4 r}{\rho R}-3\right)+\frac{2}{3}\left(\frac{4 r}{\rho R}-3\right)^{3}-\frac{1}{5}\left(\frac{4 r}{\rho R}-3\right)^{5}\right\} & \text { in } \bar{B}\left(\frac{\rho R}{2} ; \rho R\right), \\ 0 & \text { in } \Omega \backslash \bar{B}(\rho R),\end{cases}
$$

where $\rho$ is a parameter in $(0,2]$ and $R \leq \delta / 2$ is a fixed number.

Remark 2.1 It is easy to check that $\eta_{\rho} \in C^{2}(\Omega)$ satisfies the following inequalities

$$
\left|\eta_{\rho}\right| \leq 1, \quad\left|\partial_{r} \eta_{\rho}\right| \leq C \rho^{-1}, \quad \text { and } \quad\left|\partial_{r r} \eta_{\rho}\right| \leq C \rho^{-2}
$$

where $\partial_{r}$ and $\partial_{r r}$ denote the respective first and second order partial differential operators with respect to $r$. Similarly, $\partial_{\theta}$ and $\partial_{\theta \theta}$ are the partial differential operators with respect to $\theta$. Here and thereafter, we use $C$ with or without subscripts in this paper to denote a generic positive constant, possibly different at different occurrences, that is independent of $\rho$ and the mesh size $h$ introduced in subsequent sections but may depend on the domain $\Omega$.

Remark 2.2 The singular functions $s_{l}$ and the dual singular functions $s_{-l}$, both given in (2.9), are defined in each subdomain $\Omega_{j}$ and infinitely differentiable there. However, their values and derivatives may not be defined on the interfaces $\Gamma_{i j}=\partial \Omega_{i} \cap \partial \Omega_{j}$, so we need to notify that some of the inner products or norms of functions containing such singular functions or their derivatives are the summation of their values on each subdomain $\Omega_{j}$. For that reason we indicate the situation by using subindex $\Omega_{\Sigma}$ as follows;

$$
\left(\mathrm{a} \Delta\left(\eta_{\rho} s_{k}\right), \eta_{2} s_{-l}\right) \Omega_{\Omega_{\Sigma}}=\sum_{j}\left(\mathrm{a}_{j} \Delta\left(\eta_{\rho} s_{k}\right), \eta_{2} s_{-l}\right)_{\Omega_{j}}
$$

and

$$
\left\|\Delta\left(\eta_{2} s_{-l}\right)\right\|_{\Omega_{\Sigma}}^{2}=\sum_{j}\left\|\Delta\left(\eta_{2} s_{-l}\right)\right\|_{\Omega_{j}}^{2} .
$$

Similarly, we use the subindex to emphasize the smaller supports in the norms and inner products as in $\left\|\Delta\left(\eta_{2} s_{-l}\right)\right\|_{B(R ; 2 R)}$ or $\left(\mathrm{a} \phi, \Delta\left(\eta_{2} s_{-l}\right)\right)_{B(R ; 2 R)}$ in the section 3 and thereafter. Although we use these subindices to reduce the possible confusion or to emphasize the smaller supports but will omit or use only one of them to avoid possible confusion by the overuses, if necessary ([5]).

Using the cut-off function defined above, the solution of (2.2) has the following singular function representation

$$
u=w+\sum_{l=1}^{L} \kappa_{l} \eta_{\rho} s_{l}
$$


where $w \in H^{2}\left(\Omega_{j}\right)$ for $1 \leq j \leq J$ is the regular part of the solution and $\kappa_{l}$ for $1 \leq l \leq L$ is the so-called stress intensity factors. The stress intensity factors can be expressed in terms of the following extraction formula (see, e.g., [5]):

$$
\kappa_{l}=\frac{1}{2 \alpha_{l}} \sum_{i=1}^{I} \int_{\Omega_{m_{i}}}\left[f \eta_{\rho} s_{-l}+\mathrm{a}_{m_{i}} u \Delta\left(\eta_{\rho} s_{-l}\right)\right] d x
$$

where $\Omega_{m_{i}}$ are the subdomains sharing $p$ as a common vertex. Moreover, the following regularity estimate holds

$$
\sum_{l=1}^{L}\left|\kappa_{l}\right|+\sum_{j=1}^{J}\|w\|_{H^{2}\left(\Omega_{j}\right)} \leq C\|f\|_{L^{2}(\Omega)} .
$$

We end this section with a modification of Green's theorem for the regular part of the solution.

Lemma 2.1 Let $w \in H^{2}\left(\Omega_{j}\right)$ be the regular part of the solution in (2.15), then

$$
\sum_{j} \int_{\Omega_{j}} \mathrm{a}_{j} \nabla w \cdot \nabla v d x=-\sum_{j} \int_{\Omega_{j}}\left(\nabla \cdot \mathrm{a}_{j} \nabla w\right) v d x
$$

for any $v \in H_{0}^{1}(\Omega)$.

Proof: On each subdomain $\Omega_{j}$, since $\mathrm{a}_{j}$ is a constant, it is then easy to see that

$$
\int_{\Omega_{j}} \mathrm{a}_{j} \nabla w \cdot \nabla v d x=\int_{\partial \Omega_{j}} \mathrm{a}_{j} \frac{\partial w}{\partial \mathbf{n}_{j}} v d s-\int_{\Omega_{j}}\left(\nabla \cdot \mathrm{a}_{j} \nabla w\right) v d x
$$

for $w \in H^{2}\left(\Omega_{j}\right)$ and $v \in H_{0}^{1}(\Omega)$. Note that $\eta_{\rho} s_{l}$ satisfies the interface condition in (2.3) and, hence, so does the regular part of the solution, $w$. Then summing (2.19) over $j=1, \cdots, J$ and using the interface condition for $w$, we have (2.18).

\section{Well-Posed Variational Problem for $w$}

This section is the core of the method, which derives a variational problem for the regular part of the solution and proves its well-posedness. The key step of the derivation is to establish a new extraction formula for the stress intensity factors in terms of $w$ (see Lemma 3.1).

Lemma 3.1 The stress intensity factors $\kappa_{l}$ for $1 \leq l \leq L$ can be expressed in terms of $w$ corresponding to $\rho \leq 1$ by the following extraction formula

$$
\kappa_{l}=\frac{1}{2 \alpha_{l}} \sum_{i=1}^{I} \int_{\Omega_{m_{i}}}\left[f \eta_{2} s_{-l}+\mathrm{a}_{m_{i}} w \Delta\left(\eta_{2} s_{-l}\right)\right] d x,
$$

where $\Omega_{m_{i}}$ are the subdomains sharing $p$ as a common vertex. 
Proof: On each $\Omega_{j}$, applying $-\mathrm{a} \Delta$ to the equation in (2.15) with $\rho \leq 1$ and using the first equation in (1.1) give

$$
f=-\mathrm{a} \Delta w-\sum_{k=1}^{L} \kappa_{k} \mathrm{a} \Delta\left(\eta_{\rho} s_{k}\right), \quad \text { on each } \Omega_{j}
$$

Multiplying by $\eta_{2} s_{-l}$, integrating over the each subdomain $\Omega_{j}$ and summation yield

$$
\left(f, \eta_{2} s_{-l}\right)_{\Omega_{\Sigma}}=-\left(\mathrm{a} \Delta w, \eta_{2} s_{-l}\right)_{\Omega_{\Sigma}}-\sum_{k=1}^{L} \kappa_{k}\left(\mathrm{a} \Delta\left(\eta_{\rho} s_{k}\right), \eta_{2} s_{-l}\right)_{\Omega_{\Sigma}} .
$$

Similar arguments as the proof of Lemma 2.2 in [6] to each subdomains $\Omega_{j}$ and their sum leads to

$$
\left(\mathrm{a} \Delta w, \eta_{2} s_{-l}\right)_{\Omega_{\Sigma}}=\left(\mathrm{a} w, \Delta\left(\eta_{2} s_{-l}\right)\right)_{\Omega_{\Sigma}}
$$

since the diffusion coefficient $a$ is piecewise constant and the dual singular function $s_{-l}$ and $w$ satisfy the interface conditions by (2.12) and the comment in the proof of Lemma 2.1.

Now, to establish the extraction formula in (3.1) it suffices to show that

$$
\left(\mathrm{a} \Delta\left(\eta_{\rho} s_{k}\right), \eta_{2} s_{-l}\right)_{\Omega_{\Sigma}}=-2 \alpha_{l} \delta_{l k}
$$

where $\delta_{l k}$ is the Kronecker delta. To this end, a straightforward calculation using (2.4) gives

$$
\Delta\left(\eta_{\rho} s_{k}\right)=\Delta\left(\eta_{\rho} r^{\alpha_{k}} \Theta_{k}(\theta)\right)=\left(\left(2 \alpha_{k}+1\right) r^{\alpha_{k}-1} \partial_{r} \eta_{\rho}+r^{\alpha_{k}} \partial_{r r} \eta_{\rho}\right) \Theta_{k}(\theta) .
$$

It then follows from properties of the dual singular function and the cut-off functions, the fact that $\eta_{2}=1$ in $\left[\frac{\rho R}{2}, \rho R\right]$ for $\rho \leq 1$, and the orthogonality property in (2.8) that

$$
\begin{aligned}
& \left(\mathrm{a} \Delta\left(\eta_{\rho} s_{k}\right), \eta_{2} s_{-l}\right)_{\Omega_{\Sigma}} \\
= & \int_{\omega_{0}}^{\omega_{I}} \int_{\frac{\rho R}{2}}^{\rho R} \mathrm{a}\left(\left(2 \alpha_{k}+1\right) r^{-1} \partial_{r} \eta_{\rho}+\partial_{r r} \eta_{\rho}\right) r^{\alpha_{k}-\alpha_{l}} \Theta_{k}(\theta) \Theta_{l}(\theta) r d r d \theta \\
= & \int_{\frac{\rho R}{2}}^{\rho R}\left(\left(2 \alpha_{k}+1\right) \partial_{r} \eta_{\rho}+r \partial_{r r} \eta_{\rho}\right) r^{\alpha_{k}-\alpha_{l}} d r \int_{\omega_{0}}^{\omega_{I}} \mathrm{a} \Theta_{k}(\theta) \Theta_{l}(\theta) d \theta \\
= & \delta_{l k} \int_{\frac{\rho R}{2}}^{\rho R}\left(\left(2 \alpha_{k}+1\right) \partial_{r} \eta_{\rho}+r \partial_{r r} \eta_{\rho}\right) r^{\alpha_{k}-\alpha_{l}} d r,
\end{aligned}
$$

which implies (3.2) for $k \neq l$. For $k=l,(3.2)$ follows from the fact that

$$
\int_{\frac{\rho R}{2}}^{\rho R}\left[\left(2 \alpha_{l}+1\right) \partial_{r} \eta_{\rho}+r \partial_{r r} \eta_{\rho}\right] d r=\left[2 \alpha_{l} \eta_{\rho}(r)+r \partial_{r} \eta_{\rho}(r)\right]_{\frac{\rho R}{2}}^{\rho R}=-2 \alpha_{l} .
$$

This completes the proof of the lemma. 
Substituting the singular function representation of $u$ in (2.15) into the model equation in (2.2), multiplying by a test function $v \in H_{0}^{1}(\Omega)$, integrating over domain $\Omega$, and using Lemma 2.1 yield

$$
\int_{\Omega} \mathrm{a} \nabla w \cdot \nabla v d x-\sum_{l=1}^{L} \kappa_{l} \int_{\Omega_{\Sigma}} \mathrm{a} \Delta\left(\eta_{\rho} s_{l}\right) \cdot v d x=\int_{\Omega} f v d x
$$

Using the extraction formula of $\kappa_{l}$ in (3.1) and regrouping terms give

$$
\begin{aligned}
\int_{\Omega} \mathrm{a} \nabla w \cdot \nabla v d x & -\sum_{l=1}^{L} \frac{1}{2 \alpha_{l}}\left(\sum_{i=1}^{I} \int_{\Omega_{m_{i}}} \mathrm{a}_{m_{i}} w \Delta\left(\eta_{2} s_{-l}\right) d x\right) \int_{\Omega_{\Sigma}} \mathrm{a} \Delta\left(\eta_{\rho} s_{l}\right) \cdot v d x \\
& =\int_{\Omega} f v d x+\sum_{l=1}^{L} \frac{1}{2 \alpha_{l}}\left(\sum_{i=1}^{I} \int_{\Omega_{m_{i}}} f \eta_{2} s_{-l} d x\right) \int_{\Omega_{\Sigma}} \mathrm{a} \Delta\left(\eta_{\rho} s_{l}\right) \cdot v d x .
\end{aligned}
$$

Define the bilinear and linear forms by

$$
a(w, v)=(\mathrm{a} \nabla w, \nabla v)-\sum_{l=1}^{L} \frac{1}{2 \alpha_{l}}\left(\mathrm{a} w, \Delta\left(\eta_{2} s_{-l}\right)\right)_{\Omega_{\Sigma}}\left(\mathrm{a} \Delta\left(\eta_{\rho} s_{l}\right), v\right)_{\Omega_{\Sigma}}
$$

and

$$
g(v)=(f, v)+\sum_{l=1}^{L} \frac{1}{2 \alpha_{l}}\left(f, \eta_{2} s_{-l}\right)\left(\mathrm{a} \Delta\left(\eta_{\rho} s_{l}\right), v\right)_{\Omega_{\Sigma}},
$$

respectively. Then the variational problem for the regular part of the solution is to find $w \in H_{0}^{1}(\Omega)$ such that

$$
a(w, v)=g(v) \quad \forall v \in H_{0}^{1}(\Omega) .
$$

Remark 3.1 Since $s_{l} \in H^{1+\alpha_{l}-\epsilon}\left(\Omega_{m_{i}}\right)$ and $s_{-l} \in H^{1-\alpha_{l}-\epsilon}\left(\Omega_{m_{i}}\right)$ for any $\epsilon>0$ and $\Delta\left(\eta_{2} s_{-l}\right)$ and $\Delta\left(\eta_{\rho} s_{l}\right)$ equal zero around the vertex $p$, the integrals in both (3.3) and (3.4) are well-defined. The second terms in the respective bilinear and linear forms provide a singular correction so that $w \in H^{2}\left(\Omega_{j}\right)$.

In the remainder of this section, we establish the well-posedness of problem (3.5) by the use of the Fredholm alternative (see, e.g., [11]) in $H_{0}^{1}(\Omega)$. To this end, note first that the singular and dual singular functions have the following forms (see [18])

$$
s_{l}=r^{\alpha_{l}}\left(c_{l i} \sin \alpha_{l} \theta+d_{l i} \cos \alpha_{l} \theta\right) \quad \text { and } \quad s_{-l}=r^{-\alpha_{l}}\left(c_{l i} \sin \alpha_{l} \theta+d_{l i} \cos \alpha_{l} \theta\right) \quad \text { in } \Omega_{m_{i}} \text {, }
$$

where coefficients $c_{l i}$ and $d_{l i}$ depend on the diffusion coefficient a. A lengthy and elementary calculation (see [6] for a similar but simpler computation) gives the following upper bounds of $\eta_{2} s_{-l}$ and $\eta_{\rho} s_{l}$ on the respective $B(R ; 2 R)$ and $B(\rho R)$. 
Lemma 3.2 For any $0<\rho \leq 1$, we have that

$$
\left\|\Delta\left(\eta_{2} s_{-l}\right)\right\|_{B(R ; 2 R)} \leq C R^{-\alpha_{l}-1}
$$

and that

$$
\left\|\eta_{\rho} s_{l}\right\|_{B(\rho R)} \leq C(\rho R)^{1+\alpha_{l}} \quad \text { and } \quad\left\|\Delta\left(\eta_{\rho} s_{l}\right)\right\|_{B(\rho R)} \leq C(\rho R)^{\alpha_{l}-1} .
$$

To obtain the continuity and coercivity bounds of the bilinear form, we further need the well-known Poincaré-Friedrichs inequality

$$
\|v\| \leq C\|\nabla v\|
$$

and, particularly (see, e.g., [6]),

$$
\|v\|_{B(R ; 2 R)} \leq \frac{2 \omega R}{\pi}\|\nabla v\|
$$

for any $v \in H_{0}^{1}(\Omega)$.

Lemma 3.3 For $0<\rho \leq 1$, there exist positive constants $C_{0}, C_{1}$, and $C_{2}$ such that

$$
C_{0}\|\phi\|_{1}^{2} \leq a(\phi, \phi)+\frac{C_{1}}{R^{2}}\|\phi\|^{2} \quad \forall \phi \in H_{0}^{1}(\Omega)
$$

and that

$$
a(\phi, \psi) \leq C_{2}\|\phi\|_{1}\|\psi\|_{1} \quad \forall \phi, \psi \in H_{0}^{1}(\Omega) .
$$

Proof: For any $\phi$ and $\psi$ in $H_{0}^{1}(\Omega)$, it follows from the Cauchy-Schwarz inequality, Lemma 3.2 , and (3.9) that

$$
\begin{aligned}
& \left|\frac{1}{2 \alpha_{l}}\left(\mathrm{a} \phi, \Delta\left(\eta_{2} s_{-l}\right)\right)_{B(R ; 2 R)}\left(\mathrm{a} \Delta\left(\eta_{\rho} s_{l}\right), \psi\right)_{B(\rho R)}\right| \\
\leq & \frac{\overline{\mathrm{a}}^{2}}{2 \alpha_{l}}\left\|\Delta\left(\eta_{2} s_{-l}\right)\right\|_{B(R ; 2 R)}\left\|\Delta\left(\eta_{\rho} s_{l}\right)\right\|_{B(\rho R)}\|\phi\|_{B(R ; 2 R)}\|\psi\|_{B(\rho R)} \\
\leq & C \frac{\overline{\mathrm{a}}^{2}}{R^{2}} \frac{\rho^{\alpha_{l}}}{\alpha_{l}}\|\phi\|_{B(R ; 2 R)}\|\psi\|_{B(\rho R)} \leq C \frac{\overline{\mathrm{a}}^{2}}{R} \frac{\rho^{\alpha_{l}}}{\alpha_{l}}\|\phi\|\|\nabla \psi\|,
\end{aligned}
$$

where $\overline{\mathrm{a}}=\max _{1 \leq j \leq J}\left\{\mathrm{a}_{j}\right\}$. Now, the inequality in (3.11) is an immediate consequence of the Cauchy-Schwarz inequality and (3.12). Let $\underline{\mathrm{a}}=\min _{1 \leq j \leq J}\left\{\mathrm{a}_{j}\right\}$, by using $(3.12)$ with $\psi=\phi$ and the $\epsilon$-inequality, we have that, for any $\epsilon>0$

$$
\begin{aligned}
a(\phi, \phi) & \geq \underline{\mathrm{a}}\|\nabla \phi\|^{2}-C \frac{\overline{\mathrm{a}}^{2}}{R}\left(\sum_{l} \frac{\rho^{\alpha_{l}}}{\alpha_{l}}\right)\|\phi\|_{B(R ; 2 R)}\|\nabla \phi\| \\
& \geq\left(\underline{\mathrm{a}}-C \frac{\overline{\mathrm{a}}^{2}}{R}\left(\sum_{l} \frac{\rho^{\alpha_{l}}}{\alpha_{l}}\right) \epsilon\right)\|\nabla \phi\|^{2}-\frac{C \overline{\mathrm{a}}^{2}}{\epsilon R}\left(\sum_{l} \frac{\rho^{\alpha_{l}}}{\alpha_{l}}\right)\|\phi\|_{B(R ; 2 R)}^{2} .
\end{aligned}
$$


Choosing $\epsilon=\underline{\mathrm{a}}\left(C \frac{\overline{\mathrm{a}}^{2}}{R}\left(\sum_{l} \frac{\rho^{\alpha_{l}}}{\alpha_{l}}\right)\right)^{-1} / 2$ gives that

$$
a(\phi, \phi) \geq \frac{\mathrm{a}}{2}\|\nabla \phi\|^{2}-\frac{\overline{\mathrm{a}}^{4}}{\underline{\mathrm{a}} R^{2}}\left(\sum_{l} \frac{\rho^{\alpha_{l}}}{\alpha_{l}}\right)^{2}\|\phi\|^{2} .
$$

Now, (3.10) follows from the Poincaré-Friedrichs inequality in (3.8).

Now, consider the following bilinear form

$$
a_{\mu}(w, v)=a(w, v)+\mu(w, v)
$$

for $\mu \geq 0$. Let $T_{\mu}: H_{0}^{1}(\Omega) \rightarrow H^{-1}(\Omega)$ be the corresponding operator of the bilinear form $a_{\mu}(\cdot, \cdot)$, where $H^{-1}(\Omega)$ is the dual space of $H_{0}^{1}(\Omega)$ with standard dual norm denoted by $\|\cdot\|_{-1}$.

Theorem 3.1 For $0<\rho \leq 1$, we have that

(1) problem (3.5) has a unique solution $w$ in $H_{0}^{1}(\Omega)$ with $w \in H^{2}\left(\Omega_{j}\right)$ for all $j$;

(2) there exists a positive constant $\gamma$ suth that

$$
\gamma\|\phi\|_{1} \leq \sup _{\psi \in H_{0}^{1}(\Omega)} \frac{a(\phi, \psi)}{\|\psi\|_{1}}
$$

for any $\phi \in H_{0}^{1}(\Omega)$.

Proof: It follows from Lemma 3.3 that $T_{\mu}: H_{0}^{1}(\Omega) \rightarrow H^{-1}(\Omega)$ is a regular operator (i.e., it is one-to-one and onto and its inverse is bounded) for $\mu \geq C_{1} R^{-2}$ where $C_{1}$ is given in Lemma 3.3 and its Fredholm index independent of $\mu$ is zero. Hence, $T_{0}$ satisfies the Fredholm alternative: either $T_{0}$ is regular or $T_{0} w=0$ has a nontrivial solution. Since $g$ is in $H^{-1}(\Omega)$, to show the existence and uniqueness of problem (3.5), it suffices to prove that the second case does not hold. To this end, we note that

$$
a(w, v)=\left(T_{0} w, v\right)=0 \quad \forall v \in H_{0}^{1}(\Omega) .
$$

For any $v \in \mathcal{D}_{0}^{\infty}(\Omega)$, integrating by parts and using Lemma 2.1 and the interface condition in (2.11) give

$$
\begin{aligned}
0 & =a(w, v) \\
& =-(\nabla \cdot(\mathrm{a} \nabla w), v)-\sum_{l=1}^{L} \frac{1}{2 \alpha_{l}}\left(\mathrm{a} w, \Delta\left(\eta_{2} s_{-l}\right)\right)_{\Omega_{\Sigma}}\left(\nabla \cdot\left(\mathrm{a} \nabla\left(\eta_{\rho} s_{l}\right)\right), v\right) \\
& =-\left(\nabla \cdot \mathrm{a} \nabla\left\{w+\sum_{l=1}^{L} \frac{1}{2 \alpha_{l}}\left(\mathrm{a} w, \Delta\left(\eta_{2} s_{-l}\right)\right)_{\Omega_{\Sigma}}\left(\eta_{\rho} s_{l}\right)\right\}, v\right)
\end{aligned}
$$


which, together with the fact that $\mathcal{D}_{0}^{\infty}(\Omega)$ is dense in $H_{0}^{1}(\Omega)$, implies

$$
\nabla \cdot \mathrm{a} \nabla \hat{w}=0 \quad \text { in } \Omega
$$

Here, $\hat{w} \equiv w+\sum_{l=1}^{L} \frac{1}{2 \alpha_{l}}\left(\mathrm{a} w, \Delta\left(\eta_{2} s_{-l}\right)\right)\left(\eta_{\rho} s_{l}\right)$. Since $\hat{w}$ vanishes on the boundary, $\hat{w}$ is identically zero on $\bar{\Omega}$. Hence, $w$ is a linear combination of $\eta_{\rho} s_{k}$. Because $\Delta\left(\eta_{2} s_{-l}\right)=0$ on $B(R)$ and the support of $\eta_{\rho} s_{k} \subset B(R)$, we have

$$
\left(\mathrm{a} \eta_{\rho} s_{k}, \Delta\left(\eta_{2} s_{-l}\right)\right)_{\Omega_{\Sigma}}=0 \quad \forall k .
$$

This implies that $w$ is identically zero on $\bar{\Omega}$.

To show the validity of the inf-sup condition in (3.13), we use the fact that the inverse of $T_{0}$ is bounded; i.e., there exists a positive constant $\gamma$ such that

$$
\left\|T_{0}^{-1}\right\|_{H^{-1}(\Omega) \rightarrow H_{0}^{1}(\Omega)} \leq \frac{1}{\gamma}
$$

This implies that, for any $\phi \in H_{0}^{1}(\Omega)$,

$$
\gamma\|\phi\|_{1} \leq\left\|T_{0} \phi\right\|_{-1}=\sup _{\psi \in H_{0}^{1}(\Omega)} \frac{a(\phi, \psi)}{\|\psi\|_{1}}
$$

which completes the proof of (3.13) and, hence, the theorem.

Corollary 3.1 Let $w$ and $\kappa_{l}$ be the solution of (3.5) and the stress intensity factors defined in (3.1), respectively. For $0<\rho \leq 1$,

$$
u=w+\sum_{l=1}^{L} \kappa_{l} \eta_{\rho} s_{l}
$$

is the solution of (2.2).

\section{Finite Element Approximation}

This section analyzes the standard finite element approximation to $w$ based on the variational problem in (3.5). The analysis is similar to that in [6] but is more involved in details. To this end, let $\mathcal{T}_{h}$ be a partition of the domain $\Omega$ into triangular finite elements; i.e., $\Omega=\cup_{K \in \mathcal{T}_{h}} K$ with $h=\max \left\{\operatorname{diam} \mathrm{K}: \mathrm{K} \in \mathcal{T}_{\mathrm{h}}\right\}$. Assume that any $K \in \mathcal{T}_{h}$ is a subset of $\Omega_{j}$ or $K \cap \Omega_{j}=\emptyset$ and the triangulation $\mathcal{T}_{h}$ is regular. Let $V_{h}$ be continuous piecewise linear finite element space; i.e.,

$$
V_{h}=\left\{\phi_{h} \in C^{0}(\Omega):\left.\phi_{h}\right|_{K} \in P_{1}(K), \forall K \in \mathcal{T}_{h}, \phi_{h}=0 \text { on } \partial \Omega\right\} \subset H_{0}^{1}(\Omega),
$$

where $P_{1}(K)$ is the space of linear functions on $K$. It is well-known that

$$
\inf _{\phi_{h} \in V_{h}}\left(\left\|\phi-\phi_{h}\right\|+h\left|\phi-\phi_{h}\right|_{1}\right) \leq C_{A} h^{1+t}\|\phi\|_{1+t, \Omega}
$$


for any $\phi \in H_{0}^{1}(\Omega) \cap H^{1+t}(\Omega)$ and $0 \leq t \leq 1$. (4.1) is also valid on the subdomain $\Omega_{j}$. The finite element approximation to $w$ is to seek $w_{h} \in V_{h}$ such that

$$
a\left(w_{h}, v\right)=g(v) \quad \forall v \in V_{h} .
$$

To establish the $L^{2}$ norm error bound, we employ the standard duality argument. However, the standard choice of the adjoint problem does not yield an upper bound of the $L^{2}$ norm error in terms of the corresponding bilinear form. Instead, we choose the following adjoint problem with a simplified right-hand side

$$
a(v, z)=\left(w-w_{h}, v\right) \quad \forall v \in H_{0}^{1}(\Omega) .
$$

Lemma 4.1 For $0<\rho \leq 1$, problem (4.3) has a unique solution $z$ in $H_{0}^{1}(\Omega)$. Moreover, the solution $z$ is in $H^{1+\alpha_{1}-\varepsilon}(\Omega)$ for any $\varepsilon>0$ and satisfies the regularity estimate

$$
\|z\|_{1+\alpha_{1}-\varepsilon} \leq C\left\|w-w_{h}\right\| .
$$

Proof: Similar proof as that of Theorem 3.1 shows that the adjoint problem in (4.3) has a unique solution in $H_{0}^{1}(\Omega)$ and that there exists a positive constant $\gamma^{\prime}$ such that

$$
\gamma^{\prime}\|\psi\|_{1} \leq \sup _{\phi \in H_{0}^{1}(\Omega)} \frac{a(\phi, \psi)}{\|\phi\|_{1}} \quad \forall \psi \in H_{0}^{1}(\Omega)
$$

Let $z$ be the solution of (4.3), by the Cauchy-Schwarz inequality we then have that

$$
\|z\|_{1} \leq \frac{1}{\gamma^{\prime}} \sup _{\phi \in H_{0}^{1}(\Omega)} \frac{a(\phi, z)}{\|\phi\|_{1}}=\frac{1}{\gamma^{\prime}} \sup _{\phi \in H_{0}^{1}(\Omega)} \frac{\left(w-w_{h}, \phi\right)}{\|\phi\|_{1}} \leq \frac{1}{\gamma^{\prime}}\left\|w-w_{h}\right\| .
$$

It is easy to check that the solution, $z \in H_{0}^{1}(\Omega)$, of problem (4.3) satisfies

$$
\nabla \cdot \mathrm{a} \nabla z=-\sum_{l} \frac{\mathrm{a}}{2 \alpha_{l}}\left(\mathrm{a} \Delta\left(\eta_{\rho} s_{l}\right), z\right)_{\Omega_{\Sigma}} \Delta\left(\eta_{2} s_{-l}\right)-\left(w-w_{h}\right) \equiv g_{1} .
$$

Since $g_{1}$ is in $L^{2}(\Omega)$, then $z$ is in $H^{1+\alpha_{1}-\varepsilon}(\Omega)$ for any $\varepsilon>0$ and satisfies

$$
\|z\|_{1+\alpha_{1}-\varepsilon} \leq C\left\|g_{1}\right\|
$$

Now, the regularity bound in (4.4) follows from the triangle and Cauchy-Schwarz inequalities, (4.5), and Lemma 3.2 that

$$
\left\|g_{1}\right\| \leq C\left(\frac{\overline{\mathrm{a}}}{2 \alpha_{l}}\left|\left(\mathrm{a} \Delta\left(\eta_{\rho} s\right), z\right)_{B\left(\frac{\rho R}{2} ; \rho R\right)}\right|\left\|\Delta\left(\eta_{2} s_{-l}\right)\right\|_{B(R ; 2 R)}+\left\|w-w_{h}\right\|\right) \leq C\left\|w-w_{h}\right\| .
$$

This proves the inequality in (4.4) and, hence, the lemma.

The next theorem establishes error bounds of the finite element approximation in the $L^{2}$ and $H^{1}$ norms. 
Theorem 4.1 For $0<\rho \leq 1$, there exists a positive constant $h_{0}$ such that for all $h \leq h_{0}(4.2)$ has a unique solution $w_{h}$ in $V_{h}$. Moreover, let $w \in H^{2}(\Omega)$ be the solution of (3.5), then we have the following error estimates

$$
\left\|w-w_{h}\right\|_{1} \leq C h\|f\|
$$

and for any $\varepsilon>0$

$$
\left\|w-w_{h}\right\| \leq C h^{1+\alpha_{1}-\varepsilon}\|f\| .
$$

Proof: We first establish error bounds in (4.7) and (4.8) for any solution to problem (4.2) that may exist. Then, for $f \equiv 0$, the uniqueness of the solution to problem (3.5) and the error bound in (4.7) imply that $w_{h} \equiv 0$. Hence, (4.2) has a unique solution $w_{h}$ in $V_{h}$ since it is a finite dimensional problem with the same number of unknowns and equations.

To establish error bounds, note first the orthogonality property

$$
a\left(w-w_{h}, v\right)=0 \quad \forall v \in V_{h} .
$$

By choosing $v=w-w_{h}$ in equation (4.3) and using the orthogonality property in (4.9) and the continuity bound in (3.11), we have that

$$
\left\|w-w_{h}\right\|^{2}=a\left(w-w_{h}, z\right)=a\left(w-w_{h}, z-I_{h} z\right) \leq C\left\|w-w_{h}\right\|_{1}\left\|z-I_{h} z\right\|_{1}
$$

where $I_{h} z \in V_{h}$ is the nodal interpolant of $z$. By the approximation property and the regularity estimate in (4.4), we have

$$
\left\|z-I_{h} z\right\|_{1} \leq C h^{\alpha_{1}-\varepsilon}\|z\|_{1+\alpha_{1}-\varepsilon} \leq C h^{\alpha_{1}-\varepsilon}\left\|w-w_{h}\right\|
$$

which, combining with (4.10), gives

$$
\left\|w-w_{h}\right\| \leq C h^{\alpha_{1}-\varepsilon}\left\|w-w_{h}\right\|_{1} .
$$

Now, it follows from Lemma 3.3, orthogonality property (4.9), and inequality (4.11) that for any $v \in V_{h}$

$$
\begin{aligned}
C_{0}\left\|w-w_{h}\right\|_{1}^{2} & \leq a\left(w-w_{h}, w-w_{h}\right)+C_{1} R^{-2}\left\|w-w_{h}\right\|^{2} \\
& =a\left(w-w_{h}, w-v\right)+C_{1} R^{-2}\left\|w-w_{h}\right\|^{2} \\
& \leq C\left\|w-w_{h}\right\|_{1}\|w-v\|_{1}+C R^{-2} h^{2\left(\alpha_{1}-\varepsilon\right)}\left\|w-w_{h}\right\|_{1}^{2},
\end{aligned}
$$

which, together with (4.1) and (2.17), implies the validity of error bound (4.7) for sufficiently small $h$. Error bound (4.8) is a direct consequence of (4.11) and (4.7). This completes the proof of the theorem.

Remark 4.1 By using a delicate analysis as in [6], the $\varepsilon$ in the $L^{2}$ norm estimate can be removed

$$
\left\|w-w_{h}\right\| \leq C h^{1+\alpha_{1}}\|f\|
$$


Remark 4.2 Approximations to the stress intensity factors and the solution of (2.2) can be computed according to (3.1) and (3.14) as follows

$$
\kappa_{l, h}=\frac{1}{2 \alpha_{l}}\left(\mathrm{a} w_{h}, \Delta\left(\eta_{2} s_{-l}\right)\right)_{B(R ; 2 R)}+\frac{1}{2 \alpha_{l}}\left(f, \eta_{2} s_{-l}\right)_{B(2 R)}
$$

and

$$
u_{h}=w_{h}+\sum_{l} \kappa_{l, h} \eta_{\rho} s_{l},
$$

respectively. It is easy to show that

$$
\left|\kappa_{l}-\kappa_{l, h}\right| \leq C h^{1+\alpha_{1}}\|f\|
$$

for all $l$ and that

$$
\left\|u-u_{h}\right\|_{1} \leq C h\|f\| \quad \text { and } \quad\left\|u-u_{h}\right\| \leq C h^{1+\alpha_{1}}\|f\| .
$$

Remark 4.3 The system of algebraic equations arising from (4.2) consists of the standard coefficient matrix from the diffusion operator and a perturbation from the integral terms which are only nonzero on a strip away from the interface vertex. The perturbation is rank one and the algebraic system can be solved by the Sherman-Morrison formula or the standard multigrid method applied to nonsymmetric problems (see [7]).

\section{$5 \quad$ Numerical Experiment}

Since the variational problem in (3.5) for $w$ is not a standard elliptic problem, the error estimates on the finite element approximation developed in the previous section are quite limited. For example, it requires the mesh size to be sufficiently small and the $L^{2}$ norm error bound seems pessimistic. The purpose of this section is to present numerical results that either conform to the theory or show numerically that those limitations are probably not real.

The first numerical test is on an interface problem with a corner singular point. Consider the $\Gamma$-shaped domain with vertices $(1,1),(-1,1),(-1,-1),(0,-1),(0,0)$, and $(1,0)$, and partition the domain into three squares $\Omega_{1}, \Omega_{2}$, and $\Omega_{3}$ as depicted in Fig.1. Let the diffusion coefficient a $(x)$ be piecewise constant, i.e., $\mathrm{a}(x)=\mathrm{a}_{i}$ on $\Omega_{i}$, taking values

$$
\mathrm{a}_{1}=\mathrm{a}_{3}=1 \quad \text { and } \quad \mathrm{a}_{2}=100 .
$$

Then the corresponding interface problem (1.1) has only one interface vertex at the origin, and its only singular function has the form of

$$
s=r^{\alpha} \Theta(\theta)
$$


$(1,1)$
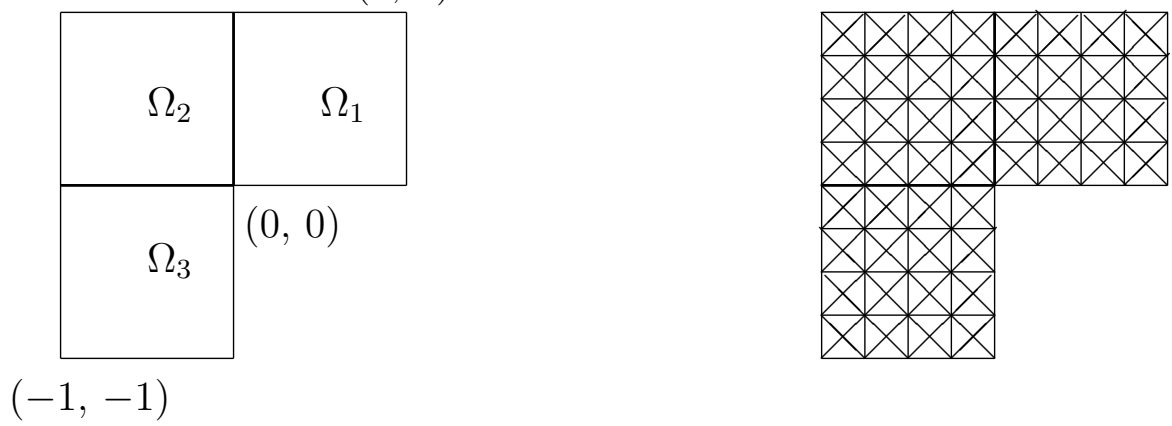

Figure 1: A $\Gamma$-shaped domain partitioned into three squares and its partitions.

with $\alpha=0.089658901772145 \ldots$ and $\Theta(\theta)=C_{i} \sin (\alpha \theta)+D_{i} \cos (\alpha \theta)$ on $\Omega_{i}$ for $i=1,2,3$. Let $\eta_{2}$ be the cut-off function defined in Section 2 with $R=1 / 8$. Choose the right-hand side function in $(2.2)$ to be

$$
f=-\mathrm{a}_{i}\left(-\frac{6}{\mathrm{a}_{i}} x\left(y^{2}-y^{4}\right)+\frac{1}{\mathrm{a}_{i}}\left(x-x^{3}\right)\left(2-12 y^{2}\right)+\Delta\left(\eta_{2} s\right)\right) \quad \text { on } \Omega_{i}
$$

so that the exact solution is

$$
u=w_{\rho}+\eta_{\rho} s,
$$

where $\eta_{\rho}$ is the cut-off function with $R=1 / 8$ and $0<\rho \leq 1$ and $w_{\rho}$ is the regular part of the solution having the form of

$$
w_{\rho}=\frac{1}{\mathrm{a}_{i}}\left(x-x^{3}\right)\left(y^{2}-y^{4}\right)+\left(\eta_{2}-\eta_{\rho}\right) s \quad \text { on } \Omega_{i}
$$

for $i=1,2,3$.

Table 1 reports numerical results of the discretization error of the regular part of the solution in the respective $L^{2}, L^{\infty}$, and $H^{1}$ norms for $\rho=1$. Even though the errors change with respect to various values of $\rho$, the difference is insignificant. It seems that the finite element approximation is not restricted to the small mesh size. As predicted in the theory, the method achieves the optimal accuracy, $O(h)$, in the $H^{1}$ norm. The order of the accuracy in the $L^{2}$ norm established in Theorem 4.1 for this example is less than 1.1. But numerical results depicted in all these three tables clearly show that it is most likely to be around 2 despite some oscillation. We also report numerical results on the approximation to the stress intensity factor. 


\begin{tabular}{||c||c||c||c||c||c||c||}
\hline \multicolumn{1}{|c||}{$h$} & $2^{-5}$ & $2^{-6}$ & $2^{-7}$ & $2^{-8}$ & $2^{-9}$ & $2^{-10}$ \\
\hline \hline \multirow{2}{*}{$L^{2}$} & $5.0549 \mathrm{e}-03$ & $1.8321 \mathrm{e}-03$ & $4.8524 \mathrm{e}-04$ & $1.1252 \mathrm{e}-04$ & $2.1761 \mathrm{e}-05$ & $5.84033 \mathrm{e}-06$ \\
\cline { 2 - 7 } & Order & 1.4642 & 1.9167 & 2.1085 & 2.3704 & 1.897623 \\
\hline \hline \multirow{2}{*}{$L^{\infty}$} & $3.2061 \mathrm{e}-02$ & $1.9981 \mathrm{e}-02$ & $1.4292 \mathrm{e}-03$ & $1.2309 \mathrm{e}-03$ & $1.5529 \mathrm{e}-04$ & $6.32335 \mathrm{e}-05$ \\
\cline { 2 - 7 } & Order & 0.6822 & 3.8053 & 0.2156 & 2.9866 & 1.296204 \\
\hline \hline \multirow{2}{*}{$H^{1}$} & $1.2451 \mathrm{e}-01$ & $6.1012 \mathrm{e}-02$ & $1.7750 \mathrm{e}-02$ & $9.1545 \mathrm{e}-03$ & $4.2923 \mathrm{e}-03$ & $2.1391 \mathrm{e}-03$ \\
\cline { 2 - 7 } & Order & 1.0291 & 1.7812 & 0.9552 & 1.0927 & 1.004734 \\
\hline \multirow{2}{*}{$\kappa$} & 1.8632 & 1.3415 & 1.0399 & 1.0207 & 1.0035 & 1.00111 \\
\cline { 2 - 7 } & Order & 1.337809 & 3.0097425 & 0.946758 & 2.564204 & 1.656795 \\
\hline
\end{tabular}

TABLE 1. Discretization Error for $\rho=1.0$
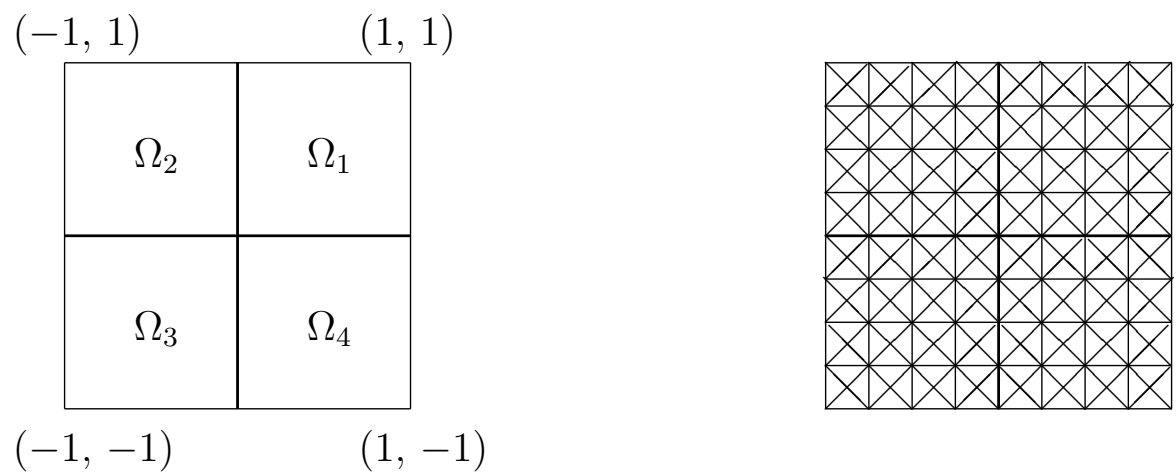

Figure 2: A square partitioned into four squares.

Similar observations as above hold true for the interface problem having interior interface points, which is presented in the next example. Consider an interface problem defined on a square $[-1,1] \times[-1,1]$ with an interior singular point at the origin. Let the domain be partitioned into four squares $\Omega_{1}, \Omega_{2}, \Omega_{3}$, and $\Omega_{4}$ as depicted in Fig.2 and let the piecewise constant diffusion coefficient $\mathrm{a}(x)=\mathrm{a}_{i}$ on $\Omega_{i}$ take values

$$
\mathrm{a}_{1}=\mathrm{a}_{3}=1, \quad \mathrm{a}_{2}=100, \quad \text { and } \quad \mathrm{a}_{4}=200 .
$$

Then this problem has only one interface vertex at the origin, and its only singular function has the form of

$$
s=r^{\alpha} \Theta(\theta),
$$

with $\alpha=0.109946076427188 \ldots$ and $\Theta(\theta)=C_{i} \sin (\alpha \theta)+D_{i} \cos (\alpha \theta)$ on $\Omega_{i}(i=1,2,3,4)$. Let $\eta_{2}$ and $\eta_{\rho}$ be the same cut-off functions as in the previous example and choose the right-hand side function in (2.2) to be

$$
f=-\mathrm{a}_{i}\left(-\frac{6}{\mathrm{a}_{i}} x\left(y^{2}-y^{4}\right)+\frac{1}{\mathrm{a}_{i}}\left(x-x^{3}\right)\left(2-12 y^{2}\right)+\Delta\left(\eta_{2} s\right)\right) \quad \text { on } \Omega_{i}
$$


so that the exact solution has the form of

$$
u=w_{\rho}+\eta_{\rho} s
$$

where $w_{\rho}$ is the regular part of the solution having the form of

$$
w_{\rho}=\frac{1}{\mathrm{a}_{i}}\left(x-x^{3}\right)\left(y^{2}-y^{4}\right)+\left(\eta_{2}-\eta_{\rho}\right) s \quad \text { on } \Omega_{i}
$$

for $i=1,2,3,4$. Numerical results with $\rho=1$ are reported in Table 2 .

\begin{tabular}{||c||c||c||c||c||c||}
\hline$h$ & $2^{-5}$ & $2^{-6}$ & $2^{-7}$ & $2^{-8}$ & $2^{-9}$ \\
\hline \hline \multirow{2}{*}{$L^{2}$} & $3.4046 \mathrm{e}-03$ & $1.4409 \mathrm{e}-03$ & $3.5434 \mathrm{e}-04$ & $8.4647 \mathrm{e}-05$ & $1.50076 \mathrm{e}-05$ \\
\cline { 2 - 6 } & Order & 1.240520 & 2.023764 & 2.065624 & 2.495766 \\
\hline \hline \multirow{2}{*}{$L^{\infty}$} & $2.3212 \mathrm{e}-02$ & $1.4278 \mathrm{e}-02$ & $1.0331 \mathrm{e}-03$ & $8.7804 \mathrm{e}-04$ & $1.1126 \mathrm{e}-04$ \\
\cline { 2 - 6 } & Order & 0.701081 & 3.788762 & 0.234623 & 2.980337 \\
\hline \multirow{2}{*}{$H^{1}$} & $9.8171 \mathrm{e}-02$ & $4.9687 \mathrm{e}-02$ & $1.4708 \mathrm{e}-02$ & $7.6175 \mathrm{e}-03$ & $3.5761 \mathrm{e}-03$ \\
\cline { 2 - 6 } & Order & 0.982431 & 1.756231 & 0.949243 & 1.090958 \\
\hline \multirow{2}{*}{$\kappa$} & 1.77842 & 1.29826 & 1.03691 & 1.01819 & 1.00316 \\
\cline { 2 - 6 } & Order & 1.383978 & 3.014487 & 1.020866 & 2.525149 \\
\hline
\end{tabular}

TABle 2. Discretization Error for interface $\rho=1$

\section{References}

[1] I. Babuska, R.B. Kellogg, and J. Pitkaranta, Direct and inverse error estimates for finite elements with mesh refinements, Numer. Math., 33 (1979), 447-471.

[2] H. Blum And M. Dobrowolski, On finite element methods for elliptic equations on domains with corners, Computing, 28 (1982), 53-63.

[3] M. Bourlard, M. Dauge, M.-S. Lubuma, and S. Nicaise, Coefficients of the singularities for elliptic boundary value problems on domains with conical points III. Finite element methods on polygonal domains, SIAM Numer. Anal., 29 (1992), 136-155.

[4] S. C. BREnNeR, Multigrid methods for the computation of singular solutions and stress intensity factor I: Corner singularities, Math. Comp., 68 (226), (1999), 559-583.

[5] S. C. Brenner And L.-Y. Sung, Multigrid methods for the computation of singular solutions and stress intensity factors III: Interface singularities, Comput. Methods Appl. Mech. Engrg. 192(2003), 4687-4702. 
[6] Z. CAI AND S.C. KIM, A finite element method using singular functions for the Poisson equation: Corner singularities, SIAM J. Numer. Anal., 39:(2001), 286299.

[7] Z. CAI, S. Kim, And B.-C. Shin, Solution methods for the Poisson equation: Corner singularities, SIAM J. Sci. Comput., SIAM J. SCI. COMPUT., 23:(2001), 672-682.

[8] M. DjaouA, Equations Intégrales pour un Probleme Singulier dans le Plan, These de Troisieme Cycle, Universite Pierre et Marie Curie, Paris, 1977.

[9] M. Dobrowolski, Numerical Approximation of Elliptic Interface and Corner Problems, Habilitationsschrift, Bonn, 1981.

[10] G. J. Fix, S. Gulati, And G. I. WakofF, On the use of singular functions with finite elements approximations, J. Comput. Phy., 13 (1973), 209-228.

[11] D. Gilbarg and N. S. Trudinger, Elliptic Partial Differential Equations of Second Order, 2nd ed., Springer-Verlag, Berlin, 1983.

[12] P. Grisvard, Elliptic Problems in Nonsmooth Domains, Pitman, Boston, MA, 1985.

[13] R.B. KellogG, Singularities in interface problems, in: B. Hubbard(ED.), Numerical Solution of Partial Differential Equations II, Academic Press, New York, (1971) 351-400.

[14] R.B. KellogG, On the Poisson equation with intersecting interfaces, Appl. Anal. 4(1975) 101-129.

[15] D. Mercier, Minimal regularity of the solutions of solutions of some transmission problems, Technical Report 01.7, Universite de Valenciennes et du HainautCambresis, 2001.

[16] S. Nicaise, Polygonal Interface Problems, Peter Lang, Frankfurt am Main, 1993.

[17] S. Nicaise, Anna-Margarete Sandig, General Interface Problems-II, Math. Methods. Appl. Aci. 17(1994) 431-450.

[18] M. Petzoldt, Regularity results for Laplace interface problems in two dimensions, J. Anal. Appl., 20:2 (2001), 1-26.

[19] A. Schatz And L. Wahlbin, Maximum norm estimates in the finite element method on plane polygonal domains, Part 1, Math. Comp., 32 (141), (1978) 73-109; Part 2 (refinements), Math. Comp., 33 (146), (1979) 465-492.

[20] Ch. Schwab, p- and hp-Finite Element Methods, Oxford University Press, Oxford, 1998. 
[21] B. A. Szabó and I. Babuska, Finite Element Analysis, John Wiley \& Sons, New York, 1991. 